\title{
OpenMethods: a compass for a more open Digital Humanities
}

\author{
Gimena del Rio Riande ${ }^{1}$, Erzsébet Tóth-Czifra ${ }^{2}$, Ulrike Wuttke ${ }^{3}$, Yoann Moranville ${ }^{4}$ \\ 1 CONICET; gdelrio@conicet.gov.ar \\ 2 DARIAH-EU; erzsebet.toth-czifra@dariah.eu \\ 3 University of Applied Sciences Potsdam; Wuttke@fh-potsdam.de \\ 4 DARIAH-EU; yoann.moranville@dariah.eu \\ * Correspondence: gdelrio@conicet.gov.ar
}

\begin{abstract}
The digital transformation has initiated a paradigm shift in research and scholarly communication practices towards a more open scholarly culture. Although this transformation is slowly happening in the Digital Humanities field, open is not yet default. The article introduces the OpenMethods metablog, a community platform that highlights open research methods, tools, and practices within the context of the Digital Humanities by republishing open access content around methods and tools in various formats and languages. It also describes the platform's technical infrastructure based on its requirements and main functionalities, and especially the collaborative content sourcing and editorial workflows. The article concludes with a discussion of the potentials of the OpenMethods metablog to overcome barriers towards open practices by focusing on inclusive, community sourced information based around opening up research processes and the challenges that need to be overcome to achieve its goals.
\end{abstract}

Keywords: Open Research Practices, Digital Humanities, Scholarly Communication, Publication Formats, Infrastructure, Research Methodology, Research Tools.

1. The digital, the open and their synergy - a missing component from Digital Humanities?

It is now beyond question that open research practices are key values of the academy in the 21st century. Having a look at how the open research culture (open science/open scholarship) made its way from grassroot movement to global policies and research agendas, we can recognize that the Internet and the digital transformation of research are among the main drivers of this paradigm shift [1-2]. In other words, there are clear and natural synergies between the open and digital: the digital transformation is paving the way for radical innovations in how research is done and disseminated in all areas. Eliminating the barriers inherent in print culture such as limitations in reach and distribution, restrictions in page numbers and difficulties in linking source materials etc., today's technologies enable us to open up much bigger parts of our research processes for analysis and reuse than just the sum of our conclusions. As a result, research can be viewed, managed, accessed and ultimately assessed in terms of the integrity and transparency of processes, rather than only as product [3].

Despite the apparent synergies between digital scholarship and open research practices, important aspects of open scholarship such as open data or reproducibility and reusability have not yet been reflected on a broad scale in the Digital Humanities (henceforth, DH). Just to name a paradigmatic case, most of the Best Practices in Digital Humanities (Nebraska, New York University, Stanford, among others) have not focused on the benefits of openness in the choice of software, hardware, reproducibility, or methods of work and collaboration. Although there are already noteworthy publishing projects and initiatives within the Arts and Humanities domain and related 
areas, such as the Language Science Press, Linguistics in Open Access, Open Edition, or Open Library of Humanities, and also the infrastructural investments centered around the OPERAS team in Europe and the Mellon Foundation in the U.S., or the Latin American meta-publishers Scielo and Redalyc, the discourse on how to open up research processes is barely established. As Marcel Knöchelmann points out in his recent publication [4], a possible reason for this is the dominant impact of the STEM disciplines on the Open Science paradigm. As a result, firmly anchoring it in the epistemic cultures of Arts and Humanities may seem challenging or require massive cultural change. However, while certain Open Science frameworks and practices like reproducibility, publication of null results, preregistration or even the preprint culture may not translate well into Arts and Humanities research practices, one may argue that core values of Open Science-research transparency, collaborative knowledge creation, reusability, or building open source tools-, are at least implicitly and naturally present in DH research practices, even though they are not necessarily branded as instances of Open Science.

In the present article, we wish to contribute to the discourse of open research practices in DH by providing a critical assessment of the OpenMethods metablog, a community platform dedicated to make open research methods, tools, and practices that are naturally emerging from doing collaborative research in DH visible and recognized both inside and outside its disciplinary context. In our contribution, we outline how OpenMethods metablog explores solutions for more open research and communication practices in a DH context, advancing the establishment of an open research culture. First, we provide a general description of the project and how our workflow is organized in order to serve an efficient and community-driven content curation (Section 2). Second, we demonstrate how the OpenMethods platform goes beyond traditional paper-based communication practices and contributes to establishing an open research culture in the Digital Humanities (Section 3). The final section concludes with a brief discussion of potentials for further development and the impact OpenMethods has made on DH so far and potentials for further development.

\section{OpenMethods: a platform to highlight collaborative, open research practices, methods and tools in Digital Humanities}

One of the major barriers to supporting and establishing more open forms of knowledge production and dissemination probably lies in the currently predominantly incentivized forms of scholarly communication which privilege individual research practices and subsequent publication in high-impact factor journals or with high-prestige journal or book publishers that operate along traditional, paper-centric formats that do not allow giving access to data, software and other underlying components necessary to explore DH scholarship in its full depth and reuse certain elements of it for other research purposes.

This situation results in many anomalous scholarly communication episodes, which we can observe day by day: authors' witty meta-reflections on the often multi-year-long publication processes and the simultaneous advancements in their field between submission and publication, or the struggle of tenure-track candidates to fit all their scholarly production into a thick paper dossier [5]. Such examples clearly signal a need to complement the traditional publication practices such as books and journal articles with novel practices of scholarly communication that allow immediate online availability of research results, closely linked with transparent documentations of research processes and underlying digital objects, but also swift responses to current events, collaborative editing and interactivity with readers.

OpenMethods highlights content in various forms and languages about DH methods and tools on its Internet platform. As a metablog, it focuses on republishing content based on a community-rooted selection workflow. The OpenMethods landing page presents the newest republished content to the site visitors. Each post entails a short English introduction to the original post as well as a link to the source post. Visitors are also able to filter and search through older content of the site using several content enrichment and categorization efforts undergone by the 
OpenMethods team. In the following, more details about the workflow and technical infrastructure will be given.

\subsection{How the OpenMethods workflow and infrastructure supports collaborative content curation}

The OpenMethods metablog provides a platform for DH experts from around the globe to highlight Open Access content related to digital methods and tools by providing a convenient curation workflow and easy to use (re-)publishing platform aimed at bringing together various formats. OpenMethods is intentionally interdisciplinary and multilingual to facilitate a timely, global disclosure and dissemination of knowledge and to raise peer recognition for open Digital Humanities tools and practices.

The metablog approach entails that the members of the international and interdisciplinary OpenMethods Editorial Team, that currently comprises 29 DH experts from 12 countries, select already published content about DH tools and methods for republication on the OpenMethods platform. Suitable online content may be proposed either by the editors or by members of the international DH community through various channels (e.g. OpenMethods nomination tool, submitting content through a form on our website or simply tagging content to our Twitter channel). Topics of interest are descriptions and critical reviews of DH methods and tools, (re-)use cases, and practical and theoretical reflections about how and why humanities research is increasingly conducted using digital methods and tools and its ensuing implications on scholarly practices and attitudes. Once a digital resource (content) has been nominated, a member of the Editorial Team evaluates (quality check) and categorizes the proposed content in order to make it easier to identify relevant high-quality resources within the metablog (see Fig. 1). This process - that leads to the final republication on the metablog as a post-includes an enrichment of the successful nominations with category tags based on the TaDiRAH taxonomy ${ }^{1}$ and the addition a brief introduction in English to each post in which one of our Editors explains its relevance to the DH communities. The Chief Editor is responsible for supervising the overall workflow and the daily maintenance of the platform. Important aspects for the selection of the Chief Editor and the members of the Editorial Team are their fields of expertise and language skills as one aim is to be able to cover as a group the selection and curation of relevant content in various languages.

\footnotetext{
${ }^{1}$ https://www.vocabularyserver.com/tadirah/en/ (accessed 27 February 2020).
} 


\section{How does it work?}

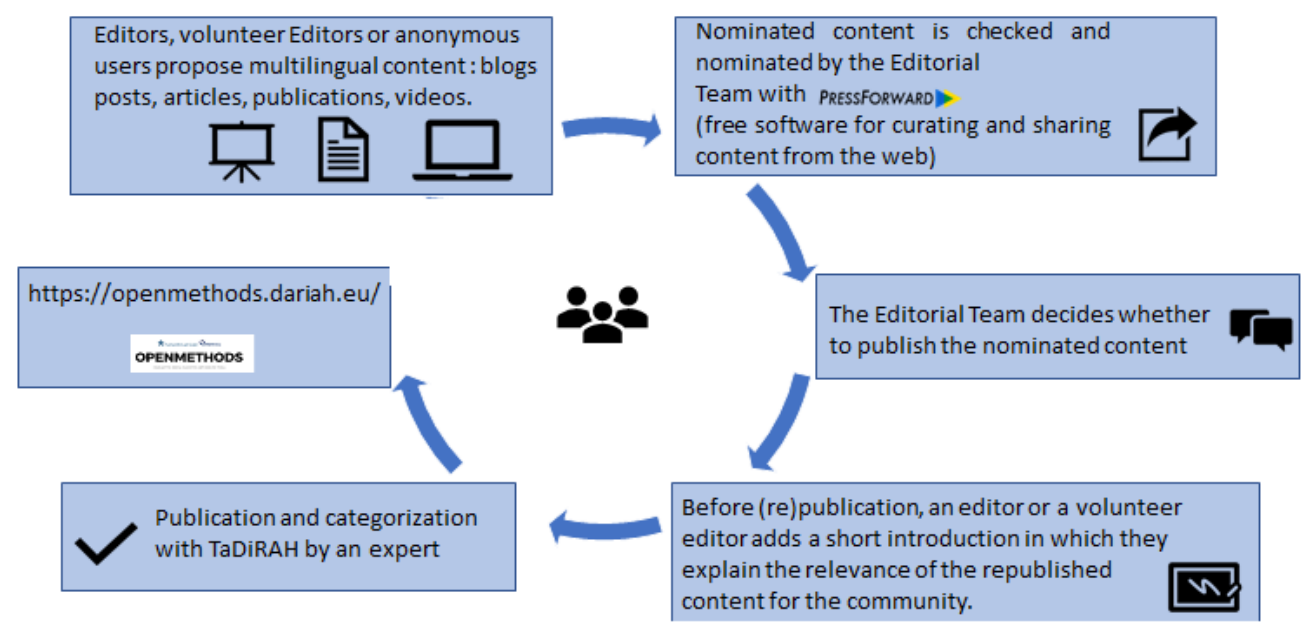

Figure 1. Description of the editorial workflow performed at OpenMethods.

The requirements for the technical infrastructure of OpenMethods were twofold: 1) it should be easy to set up and 2) it should allow for rapid, dynamic discussion around existing open access online content while offering a private curation/discussion space for the Editorial Team. Through exchange with the community and research into comparable approaches such as DHNow ${ }^{2}$, we identified a combination of a WordPress based Content Management System with the PressForward plugin ${ }^{3}$ that served these needs. PressForward has a crucial role in the efficient management of the OpenMethods editorial workflow as almost all steps of the Editorial Team's work (such as content nomination, discussion, evaluation, publishing, keeping track of published content) can be undertaken within this plugin. Recognizing its importance in our architecture, we had to make sure it was going to fit every aspect we envisioned while designing the concept of OpenMethods. With this goal in mind we contributed to the core source code of the PressForward plugin, e.g. we fixed some bugs and added some features to the tool, which had been incorporated in its main Github repository. ${ }^{4}$

$2 \quad$ http://digitalhumanitiesnow.org/ (accessed 27 February 2020).

$3 \quad$ https://pressforward.org/(accessed 27 February 2020).

$4 \quad$ https://github.com/PressForward/pressforward (accessed 27 February 2020). 


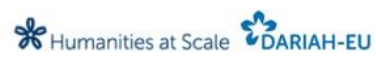 \\ OPENMETHODS}

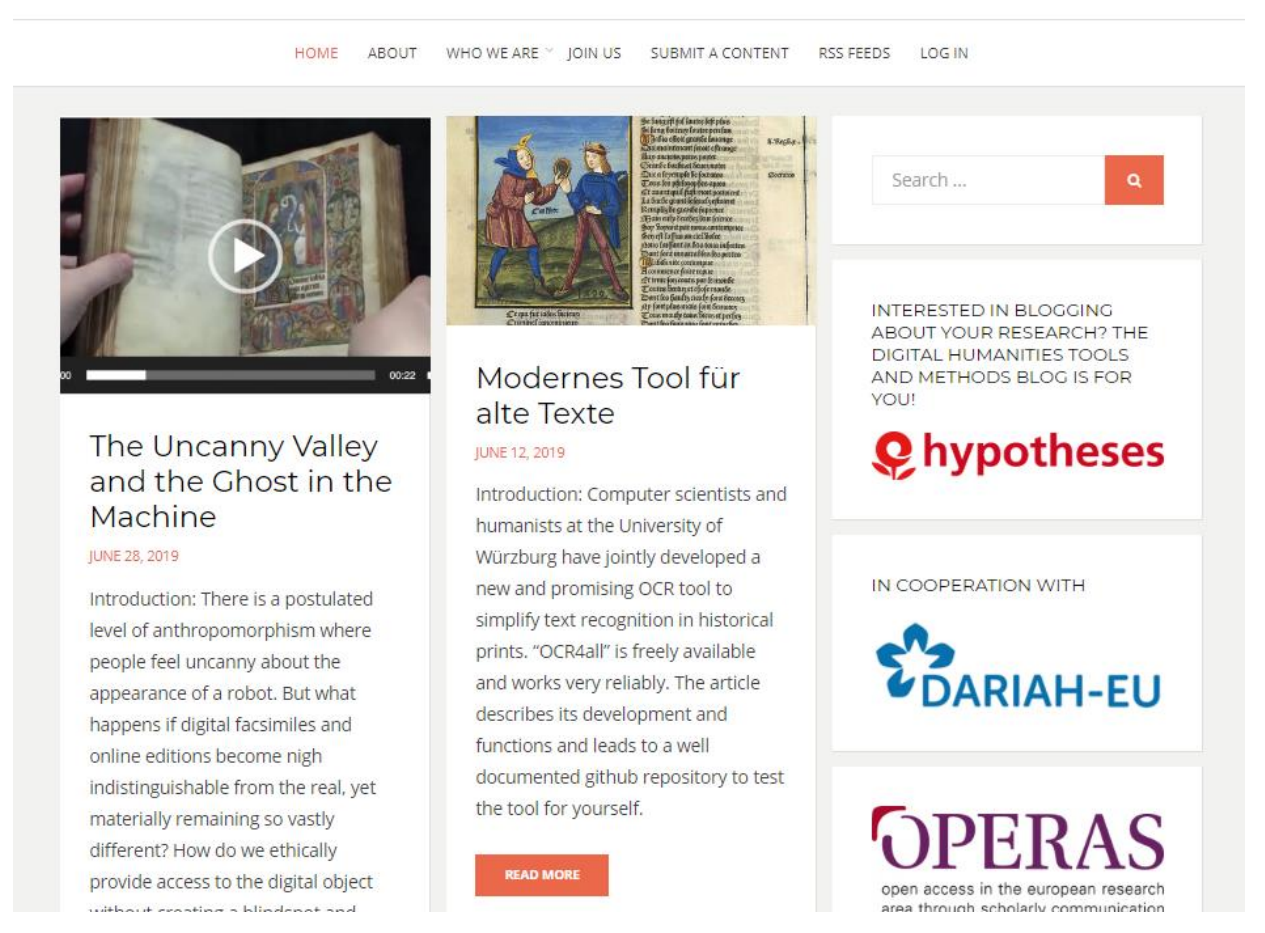

Figure 2. The OpenMethods metablog.

The OpenMethods platform has been developed in partnership with the DARIAH and OPERAS communities since it is an offspring of the DARIAH "Humanities at Scale" project [6]. Recognizing its value and the community around it, DARIAH-EU decided to provide a stable technical hosting environment and to delegate the role of Editor in Chief to a DARIAH employee. This way the sustainability of the platform is guaranteed: it operates in a $100 \%$ public environment and is not exposed to typical uncertainties around post-grant funding challenges many DH projects and digital outputs are facing today. ${ }^{5}$

\section{How OpenMethods contributes to the establishment of open research practices in Digital Humanities}

OpenMethods offers a collaborative, community-driven model of open scholarly communication that is growing out of community practices and needs in DH [7]. It goes beyond traditional paper-based communication practices and contributes to the establishment of an open research culture in $\mathrm{DH}$ in several ways, as explained below.

3.1. Focus on tools and methods as genuine research outputs and their better integration into the mainstream scholarly communication

OpenMethods is dedicated to the critical discussion of DH tools and methods. Digital tools and methods are genuine research outputs whose academic recognition is lagging behind monographs and journal articles that still serve as the highest value currency in current academia [8]. As it has been repeatedly pointed out (see e. g. [5.], [9-10]), empowering emerging evaluative cultures 
around novel digital practices and objects as scholarship is a vital step towards their inclusion into the research assessment criteria. Thus, the DH expert community forming the OpenMethods team aims to increase trust towards both open access scholarship and open good practices in DH research. OpenMethods aims not only at raising peer recognition for existing digital resources, one of its main objectives is to explicitly facilitate a culture of reuse of these materials by showcasing good practices for the adoption of existing services and their underlying software for different research questions, environments, and needs.

\subsection{Recognizing and promoting multilingualism and a greater diversity in terms of content types that have} yet to be truly incorporated into the scholarly communication ecosystem

OpenMethods is inclusive with a variety of communication formats like blog posts, videos, preprints, podcasts, lessons, etc. that are becoming increasingly important aspects of scholarly work [12] because they accelerate discussions within and outside of academia and are flexible enough to represent the dynamic and multimodal nature of the DH methodology discourse. OpenMethods' inclusiveness does not only pertain to content types that usually remain out of the scope of the traditional paradigms of scholarly communication but is also present in terms of languages. From the beginning one of our aims has been raising awareness to the value of language diversity by enhancing visibility and recognition of languages and cultures other than English, thus weakening the hegemonic position of English in the DH discourse [13]. The multilingual character of the platform gives space for the equal representation of $\mathrm{DH}$ scholarship in/from different language communities and cultures, yet currently this possibility is not exploited to its full extent. The challenges associated with keeping the multilingual character of the platforms are discussed in Section 4.

\subsection{Experimenting with a novel form of expert community review}

Trust is a central component in our modus operandi. To successfully fulfil the navigation/compass function of the platform in the increasingly noisy and dynamically evolving $\mathrm{DH}$ landscape [14], and direct our readership to content that is worth their time to read and explore, we found it important not to automate harvesting and aggregation as many aggregation/discovery platforms do. Instead, a significant portion of our editorial workflow is dedicated expert curation and filtering. Since a traditional, fully-fledged peer-review would not be compatible with another core component of the platform, timeliness and speedy/rapid communication, we put a more flexible and collaborative evaluation procedure in place. The expert community review performed on content nominations are very brief reflections on whether the nominated content is of value for the DH community, based on criteria such as consistency and comprehensiveness, clear focus, openness and reusability of the method/tool in question etc. Even such minimal reviews evolve often into a discussion among the Editorial Team and in many cases spark a lively internal exchange about hot topics in Digital Humanities methodology.

\subsection{Putting Digital Humanities tools in service of scholarly communication and more effective content discovery}

OpenMethods does not only propagate the culture of reuse but has been built on reused tools itself: the platform is based on a WordPress Content Management System and the PressForward plugin optimized for the specifications of OpenMethods. Besides, we are constantly seeking ways to put DH tools in service of more effective content discovery and enrichment. For this purpose, we have recently created plugins to achieve interoperability with the entity recognition NERD service and the research discovery platform ISIDORE to increase the visibility and discoverability of our content. 


\subsubsection{The NERD plugin 6}

This plugin allows integration of $\mathrm{NERD}^{7}$, a service that recognizes and disambiguates named entities, with WordPress. As a form of content enrichment, the NERD plugin automatically creates tags from the named entities offered by NERD when provided the full text of the original article that has been republished on OpenMethods. The tags, in return, are used to propose extra information coming from Wikipedia and Wikidata. These tags contribute to the better discoverability and searchability of content on OpenMethods and add extra context layers and therefore are especially useful for newcomers to the field.

\section{SEPTEMBER 15, 2019 - BY ROMBERT STAPEL \\ Analyzing Documents with TF-IDF | Programming Historian \\ Introduction by OpenMethods Editor (Rombert Stapel):}

The indispensable Programming Historian comes with an introduction to Term Frequency - Inverse Document Frequency (tf-idf) provided by Matthew J. Lavin. The procedure, concerned with specificity of terms in a document, has its origins in information retrieval, but can be applied as an exploratory tool, finding textual similarity, or as a pre-processing tool for machine learning. It is therefore not only useful for textual scholars, but also for historians working with large collections of text.
66 This lesson focuses on a foundational natural language processing and information retrieval method called Term Frequency - Inverse Document Frequency (tf-idf). This lesson explores the foundations of $\mathbf{t}$-idf, and will also introduce you to some of the questions and concepts of computationally oriented text analysis.

Source: Analyzing Documents with TF-IDF | Programming Historian

Share this:

(1) (f)

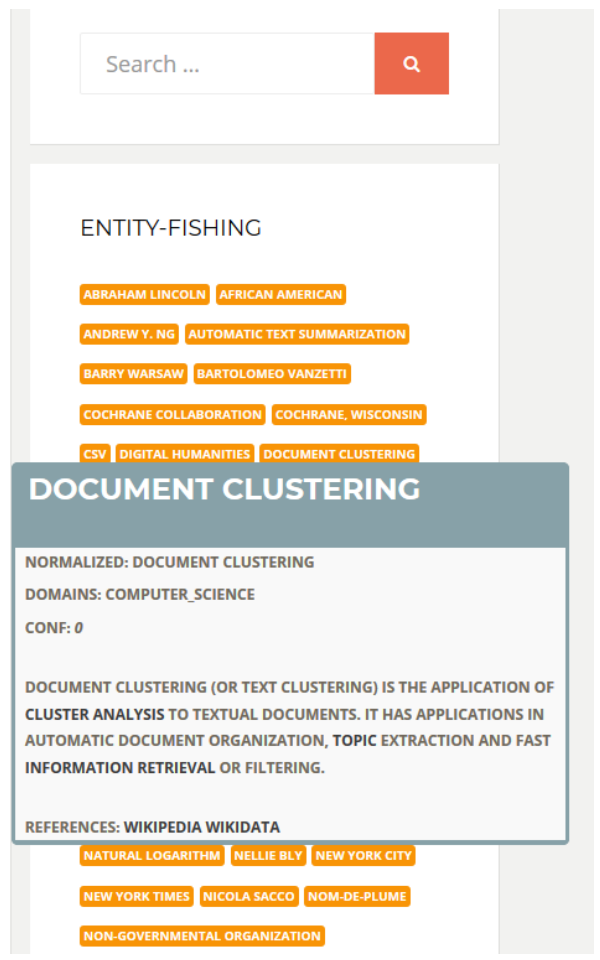

Figure 3. Automatically generated tags on OpenMethods content with links to Wikidata and Wikipedia.

\subsubsection{The enriched metadata in RDFa plugin ${ }^{8}$}

The second plugin increases the findability of our content on other discovery platforms via metadata enrichment, primarily by ISIDORE, an indexing and search service for the humanities and social sciences that serves as a single point of entry for a wide range of open SSH resources, such as data, publications and other materials. To enable harvesting and indexing our content by ISIDORE, and therefore increasing the discoverability and interoperability of our posts, OpenMethods has to be able to present information in a format understood by ISIDORE. For this purpose, we created this WordPress plugin that allows the OpenMethods team to add Dublin Core metadata enrichments in RDFa within the HTML header of each post.

$6 \quad$ https://github.com/DARIAH-ERIC/nerd-wp/ and https://wordpress.org/plugins/nerd-wp/ (accessed 27 February 2020).

$7 \quad$ https://github.com/kermitt2/entity-fishing (accessed 27 February 2020).

$8 \quad$ https://github.com/DARIAH-ERIC/rich-meta-in-rdfa/ and https://wordpress.org/plugins/rich-meta-in-rdfa/ (accessed 27 February 2020). 
Achieving interoperability with ISIDORE benefits OpenMethods in two ways: 1) connecting the platforms enables the integration of content that could not be indexed otherwise on research discovery platforms because of their language (other than English) or content type properties (e.g. blogs, videos or podcasts scattered across the web with insufficient metadata). The metadata enrichment allows us to embed such content in a large corpus of electronic publications, corpora, databases and scientific news. 2) Another benefit of the integration has to do with gaining visibility and hence recognition for our editors' work. As a result of our content being indexed by ISIDORE, each editor who contributed to the platform with introduction snippets receives an author page on ISODORE listing all their contributions with proper metadata. This allows our editors to easily report their contributions or include them into their CV or publication record.

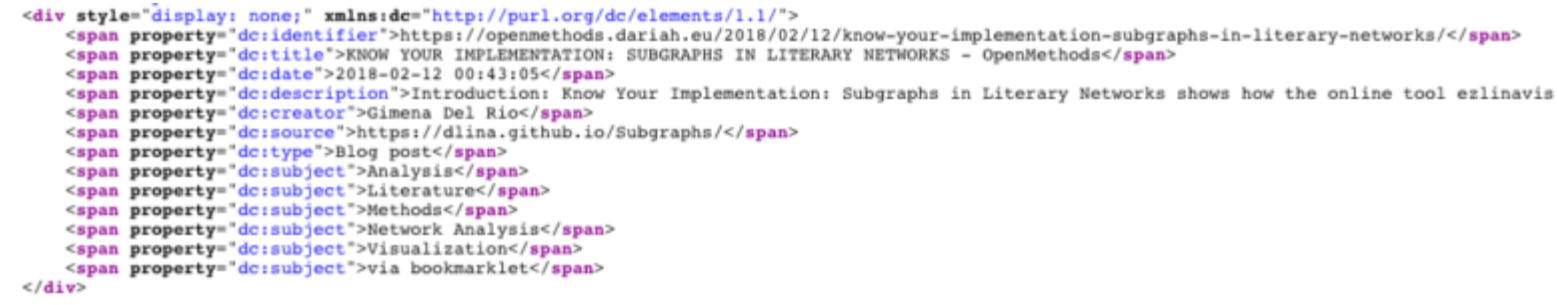

Figure 4. Dublin Core enrichment in OpenMethods' metadata.

\subsubsection{The ISIDORE Suggestion plugin (created by the Huma-Num team ${ }^{9}$ )}

To add an extra discovery layer to the OpenMethods platform, we integrated this third plugin developed by the ISIDORE team that enables the listing of similar content recommendations to OpenMethods posts.

\section{Success criteria, challenges, impact}

Our goal is to reach and engage the widest possible range of $\mathrm{DH}$ communities, from beginners who wet their toes for the first time with digital methods and tools and are curious what these have to offer and how they work, to DH experts who are shaping specific research areas as representatives for particular methods. However, achieving such a goal entails facing a range of difficulties:

- First of all, the decision of staying with manual content curation and community review to curate a white list of content about DH methods and tools has its limitations in terms of sufficient coverage of all the evolving DH discourses and the pace of publication. This could be at least partially solved by constantly growing the Editorial Team.

- On the other hand, the model of community curation offered by OpenMethods has been recognized as a model for an infrastructure building project of a much greater scale, the Social Sciences and Humanities Open Cloud (SSHOC).

- Reaching a critical number of readers as a result of focused outreach strategy. We constantly increase our readership via actively disseminating our content via our Twitter channel and presenting the platform in DH conferences, but we still need to do more.

- Finding solutions for long-term incentivisation of the editors, ensuring that editors are recognised for their contributions, and sustaining a viable pool of editors. As can be seen above, achieving interoperability with the research discovery platform ISIDORE points to this direction.

\footnotetext{
${ }^{9}$ https://www.huma-num.fr/
} 
Additionally, integrating editors and authors ORCID IDs with the platform to make their contributions more visible would be a significant step forward.

- Finally, English plays a crucial role in disseminating scientific knowledge and this language hegemony is reflected in the dominance of English-language content on our platform. Part of this challenge is that especially in the case of smaller languages such as Hungarian and Greek, the respective DH communities themselves prefer to represent their research in English. Although going against such distortions and maintaining multilingualism requires extra effort from the Editorial Team, in line with the Helsinki Initiative ${ }^{10}$ we strongly see the value in providing equal access to research and knowledge in a variety of languages. We have posts in different languages, such as Spanish, German or Polish, but we are still aiming at improving multilingualism and a global community. The translation of the landing page of the platform to multiple languages is a future improvement in this direction. Promoting multilingualism in scholarly communication is an especially important aspect in democratizing access to knowledge in the context of arts of humanities research/scholarship usually grounded in regional, national and language-specific communities.

We believe that opening up both research processes and scholarly outputs works best when it is deeply situated in the respective epistemic cultures. How are we to make claims for the value of non-traditional scholarly communication in the humanities? How can we strengthen evaluation cultures around novel forms of digital research outputs and methods so these can be truly incorporated into and recognized in the current research assessment and evaluation systems as genuine/valuable forms of scholarship? How can we better align our communication practices with our increasingly digital workflows? What are the potentials of community curation in keeping a timely discourse around a specific DH topic area alive? OpenMethods strives to tackle these questions while providing a platform and build a strong community around it to navigate humanists through the rich and dynamically evolving landscape of DH tools and methods and, at the same time, to increase the recognition of good open practices that are organically growing out of DH research realities.

\section{Author Contributions:}

Conceptualization, Gimena del Rio Riande, Erzsébet Tóth-Czifra, Ulrike Wuttke; methodology, Gimena del Rio Riande, Erzsébet Tóth-Czifra, Ulrike Wuttke; software, Yoann Moranville; writing-original draft preparation, Gimena del Rio Riande, Erzsébet Tóth-Czifra, Ulrike Wuttke, Yoann Moranville; writing-review and editing, Gimena del Rio Riande, Erzsébet Tóth-Czifra, Ulrike Wuttke; visualization, Erzsébet Tóth-Czifra; supervision, Ulrike Wuttke; project administration, Erzsébet Tóth-Czifra; funding acquisition, DARIAH-EU.

\section{References}

1. Suber, P. Open Access Overview. Focusing on Open Access to Peer-Reviewed Research Articles and their Preprints. 2006. http://legacy.earlham.edu/ peters/fos/overview.htm (accessed 27 February 2020)

2. Neylon, C. Governing Open Science. Introduction. In Contextualizing openness: situating open science; Chen, L., Okune, A., Hillyer, B., Albronz, D., Eds.; 2019. pp. 125-133.

3. Ogburn, J.L. Extending the Principles and Promises of Scholarly Communication Reform: A Chronicle and Future Glimpses. In Open access and the future of scholarly communication: policy and infrastructure; Smith, K. L., Dickson, K.L., Eds.; Rowman \& Littlefield, 2016. pp. 1-30.

4. Knöchelmann, M. Open Science in the Humanities, or: Open Humanities? Publications 2019, 7, 65.

${ }^{10}$ https://www.helsinki-initiative.org/ 
5. Eve, M.P. Violins in the Subway: Scarcity Correlations, Evaluative Cultures, and Disciplinary Authority in the Digital Humanities. In Digital Technology and the Practices of Humanities Research. Edmond, J. Ed.; Cambridge, UK: Open Book Publishers, 2020. pp. 105-122. DOI: 10.11647/OBP.0192.

6. Engelhardt, C., Leone, C., Larrousse, N., Montulio, D., y Moranville, Y., Mounier, P., Oltersdorf, J., Ribbe, P. \& Wuttke, U. Open Humanities Methods Review Journal. [Research Report] DARIAH; TGIR HumaNum (UMS 3598); Göttingen State University Library, 2017. https://hal.archives-ouvertes.fr/hal-01685852 (accessed 27 February 2020).

7. Spiro, L. This Is Why We Fight: Defining the Values of the Digital Humanities. In Debates in the Digital Humanities. : Gold, M. K. Ed.; Minnesota: University of Minnesota Press, 2012. pp. 16-35.

8. Schreibman, S., Mandell, L., \& Olsen, S. Introduction. Profession, 2011, 123-135. DOI: 10.1632/prof.2011.2011.1.123.

9. Baillot, A. A certification model for digital scholarly editions: Towards peer review-based data journals in the humanities. Preprint. 2016. https://halshs.archives-ouvertes.fr/halshs-01392880 (accessed 27 February 2020).

10. Tóth-Czifra, E. Laying the Pavement Where People Actually Walk: Thoughts on Our Chances of Bringing Scholarship Back to the Heart of Scholarly Communication. Blog post. 2019. https://dariahopen.hypotheses.org/645 (accessed 27 February 2020).

11. Ermolaev, N., Li,X., Siemens, L., \& Kaufman, M. Project Management for the Digital Humanities. Conference $\begin{array}{lllll}\text { panel at } & \text { DH2018 } & \text { conference. } & 2018 .\end{array}$ https://cdh.princeton.edu/media/uploads/documents/cdh_dh2018_pm_design_presentation.pdf (accessed 27 February 2020).

12. Dacos, M., \& Mounier, P. Les carnets de recherche en ligne, espace d'une conversation scientifique décentrée. Albin Michel. 2010.

13. Rio Riande, G., León, R., Calarco, G., \& Striker, G., Eds. Humanidades Digitales: Construcciones locales en contextos globales. Buenos Aires: Universidad de Buenos Aires. 2018.

14. Wouters, P., Beaulieu, A., Scharnhorst, A., \& Wyatt, S., Eds. Virtual knowledge: Experimenting in the Humanities and the Social Sciences. Cambridge, MA: The MIT Press. 2013. 